\title{
A development study of drain fluid gastrografin as a biomarker of anastomotic leak
}

\author{
David A. Clark ${ }^{1,2,3,4}$, Edward Yeoh ${ }^{5}$, Aleksandra Edmundson ${ }^{2,3}$, Craig Harris ${ }^{2,4}$, Andrew Stevenson ${ }^{2,3,4}$, \\ Daniel Steffens ${ }^{1}$, Michael Solomon ${ }^{1}$ \\ ${ }^{1}$ Faculty of Medicine and Health, University of Sydney and Surgical Outcomes Research Centre (SOuRCe), Sydney, NSW; ${ }^{2}$ Department of \\ Surgery, Royal Brisbane and Women's Hospital, Brisbane, QLD; ${ }^{3}$ Faculty of Medicine, University of Queensland, St Lucia, QLD; ${ }^{4}$ Department \\ of Surgery, St Vincent's Private Hospital Northside, Chermside, QLD; ${ }^{5}$ Department of Radiology, Princess Alexandra Hospital, Woolloongabba, \\ QLD, Australia
}

Purpose: Anastomotic leakage (AL) is the anathema of colorectal surgery. Its occurrence leads to increased morbidity and mortality and a prolonged hospital stay. Much work has gone into studying various biomarkers in drain fluid to facilitate early detection of AL. This stage 2 a development study aims to assess the safety and feasibility of reliably detecting the iodine in Gastrografin (GG; Bayer Australia Ltd.) in drain fluid and stool samples by dual-energy computed tomography (DECT).

Methods: This is a prospective, observational, controlled, consecutive cohort study establishing the safety and feasibility of the detection of GG in surgical drain fluid and stool as a biomarker of AL when patients with a low pelvic colorectal anastomosis undergo luminal flushing of the rectal tube with GG.

Results: Ten consecutive patients were allocated to the saline flush group and the following 10 to the GG flush group. Three patients in the saline flush group developed an AL. One patient in the GG flush group developed an AL. An elevation in the drain fluid GG was detected using DECT on the day of clinical deterioration. None of the patients in the control group were found to have a positive result on DECT.

Conclusion: This study demonstrates the safety of a novel approach to the early detection of AL from extraperitoneal colorectal anastomoses. The technique requires validation in a larger cohort and a multicenter study is planned to investigate the efficacy of GG rectal tube flushes as an early biomarker of AL in low pelvic anastomoses.

Keywords: Gastrografin; Biomarkers; Anastomotic leak; Dual-energy computed tomography; Drain fluid

\section{INTRODUCTION}

Anastomotic leakage $(\mathrm{AL})$ is the anathema of colorectal surgery. Its occurrence leads to increased morbidity and mortality and a prolonged hospital stay [1]. Much work has been devoted to the identification of patients at high risk of AL; nonetheless, low-risk

Received: Jun 15, 2020 - Revised: Dec 8, 2020 • Accepted: Dec 24, 2020 Correspondence to: David A. Clark, MBBS, FRACS, FRCS Ed

Faculty of Medicine, St Vincent's Private Hospital Northside, 627 Rode Road, Chermside, OLD 4032, Australia

Tel: +61-7-33502088, Fax: +61-7-33502333

E-mail: David.Clark@doctors.org.uk

ORCID: https://orcid.org/0000-0002-2065-3012

(C) 2022 The Korean Society of Coloproctology

This is an open-access article distributed under the terms of the Creative Commons Attribution NonCommercial License (https://creativecommons.org/licenses/by-nc/4.0) which permits unrestricted noncommercial use, distribution, and reproduction in any medium, provided the original work is properly cited. patients still suffer AL and a delay in diagnosis has a measurable effect on mortality [2].

Current approaches to the early detection of $\mathrm{AL}$ are nonspecific and insensitive. Systemic biomarkers have poor positive predictive value but the better negative predictive value and are more useful in indicating which patients are at lower risk of $\mathrm{AL}$, and thus are considered safe for discharge following surgery [3]. Drain fluid biomarkers have the potential to sample the environment around the anastomosis and have shown promise but suffer from a lack of validated specificity and cutoff values. As a consequence, $\mathrm{AL}$ is often diagnosed at an advanced stage with the presentation of clinical symptoms and often secondary complications.

Gastrografin (GG; diatrizoate, also known as amidotrizoate meglumine and sodium amidotrizoate; Bayer Australia Ltd., Pymble, NSW, Australia; the registered trademark of the Bayer Group, Germany) is a water-soluble, contrast solution commonly 
used for abdominal computed tomography (CT). In clinical practice, when administered orally or as an enema, GG acts as a radiological contrast for the detection of ALs by CT.

The iodine in GG can be measured directly and quantitated with dual-energy CT (DECT) and the protocol has been established and validated in a prior study (imaging protocol in Appendix 1) [4]. In patients who routinely have a rectal tube inserted, after a low colorectal anastomosis, this tube may be flushed with GG quarter in die (QID). Measuring the iodine in a sample of the drain fluid may serve as a biomarker of extravasation of the GG and thus a biomarker of AL of luminal contents.

The IDEAL (Idea, Development, Exploration, Assessment, Long-term study) Collaboration has set out recommendations for the introduction of new surgical techniques [5-7]. The stage 2a development studies report prospective outcomes from a single center with a small and selected sample size. They require ethics approval [8], registration of the protocol, and description of any modification to the technique as the study progresses. Outcomes reported are primarily safety but also technical and procedural success [9].

This Phase 1 , stage 2a development study aimed to assess the safety of GG rectal tube flushes and the feasibility of detecting GG in postsurgical samples by DECT.

\section{METHODS}

This is a prospective, observational, controlled, consecutive cohort, stage 2a development study establishing the safety of GG rectal tube flushes. The study also assesses the feasibility of a new methodology for the detection of GG in surgical drain fluid and stool, following a low colorectal anastomosis and without a diverting loop ileostomy. This study was based at a single private tertiary institution and with 3 participating surgeons. Patients were enrolled between November 2018 and September 2019.

Safety was assessed by recording the postoperative mortality and complications as graded by the Clavien-Dindo classification [10]. This study was prospective, signed patient consent was obtained, and the ethics approval was granted by the St Vincent's Health and Aged Care (SVHAC) Human Research Ethics Committee (No. HREC 19/01). The trial was registered with the Australian New Zealand Clinical Trials Registry (ACTRN12619001687189).

\section{Participants}

Patients with the following inclusion and exclusion criteria were invited to participate. Inclusion criteria were as follows: patients undergoing a rectal resection with an extraperitoneal anastomosis (i.e., within $10 \mathrm{~cm}$ of the anal verge) and without a diverting loop ileostomy; placement of a pelvic drain and rectal tube at surgery. The exclusion criterion is an allergy to iodine.

A sample size of 20 was chosen. This is in line with the IDEAL recommendations for a stage 2 a development study. We recruited 20 consecutive patients undergoing a rectal resection with an ex- traperitoneal anastomosis and without a diverting loop ileostomy. The first 10 patients underwent flushing of the rectal tube with normal saline ( $30 \mathrm{~mL}, \mathrm{QID})$ and served as a control group. This is the usual clinical practice in our institution, to prevent blockage of the rectal tube. The subsequent 10 patients, who were eligible for participation, had their rectal tubes flushed with GG $(30 \mathrm{~mL}$, QID). The morning flush was administered at 6:00 AM. The drain fluid was collected at 6:30 AM daily by the night shift nurses. A sample of rectal tube fluid was also collected 15 to 30 minutes after administration of the saline or GG flush to assess the baseline intraluminal iodine level.

\section{Gastrografin safety and applications}

GG is a hyperosmolar water-soluble iodinated radiological contrast media and is recorded as ARTG ID 10684 on the Australian Register of Therapeutic Goods maintained by the Therapeutic Goods Administration [11].

GG may be administered per oral or as an enema and is used routinely in clinical practice. Its safety has been established in a number of randomized trials [12]. A meta-analysis of contrast enemas found that procedures involving GG were safe with only 1 reported complication of the 1,169 procedures studied. GG and Urografin (Bayer Australia Ltd.) were the most common agents used in these radiological procedures [13]. Aside from its routine use in radiology, GG has been safely employed orally in patients with small bowel obstruction and assessed as a prokinetic in prolonged postoperative ileus $[14,15]$.

\section{Gastrografin measurement by dual-energy computed tomography}

DECT is a new technology that allows acquisition of 2 datasets from the same anatomical region at different voltages [16]. In contrast to single-spectrum imaging, which depicts the organs based on spatial distribution of the object attenuation, DECT is sensitive to the chemical composition. Hence, DECT is capable of differentiating materials with different atomic numbers. The organically bound iodine in GG is able to be simply quantitated using a DECT protocol described in Appendix 1. The lower limit of detection of a solution containing GG was at a concentration of $0.097 \%$, which correlated to an iodine density of $>1 \mathrm{mg} / \mathrm{mL}$. The upper limit of the true negative levels of GG measured in drain fluid by DECT was derived by calculating the mean $(+3$ standard deviation (SD)) iodine density $(\mathrm{mg} / \mathrm{mL})$ in GG negative samples. We have established measurements of iodine density above 1.2 $\mathrm{mg} / \mathrm{mL}$ to represent a positive result for the presence of GG in a solution measured by DECT [4].

\section{Pelvic drains}

Participating surgeons in this study routinely place a pelvic drain after a low anterior resection with an extraperitoneal anastomosis. It is possible that drains may be malpositioned or dislodged and this may lead to a false-negative result in the setting of AL detec- 
tion. While it is not intended that all patients should undergo routine imaging to confirm position, the majority of those experiencing a clinical AL will undergo CT imaging as part of usual clinical practice, presenting an opportunity for the assessment of the drain position in the pelvis.

\section{Rectal tubes}

In our institution, it is routine practice to place a 28 -gauge Foley catheter per-anally and across the sphincter in all patients undergoing a rectal resection with an extraperitoneal anastomosis. Balloons are inflated with $12 \mathrm{~mL}$ of water or sutured in place with the balloon deflated. The purpose of the rectal tube is to decompress the rectum across the sphincter and often it will sit across the anastomosis. Rectal tubes are routinely flushed with $30-\mathrm{mL}$ saline QID following surgery to prevent blockage.

\section{Sample collection \\ Measurement of drain fluid Gastrografin levels (day 1 to 5 or longer if drain remains in situ after 5 days)}

Drain fluid samples were collected from Bellovac (Wellspect HealthCare, Mölndal, Sweden) drains 15 to 30 minutes after administration of the morning rectal tube flush. The drainage bag (Fig. 1) is removed and the bellows are emptied into a sterile plastic pot. A $10-\mathrm{mL}$ sample was collected on day 1, 2, 3, 4, 5, or until drain removed for the measurement of GG by DECT. A single sample of rectal tube fluid was collected on the first postoperative day to measure the intraluminal GG level. The daily collection of drain or rectal fluid samples presents no risk, discomfort, or inconvenience to the patient.

\section{Data collection}

Consecutive, eligible patients provided written consent for inclu-

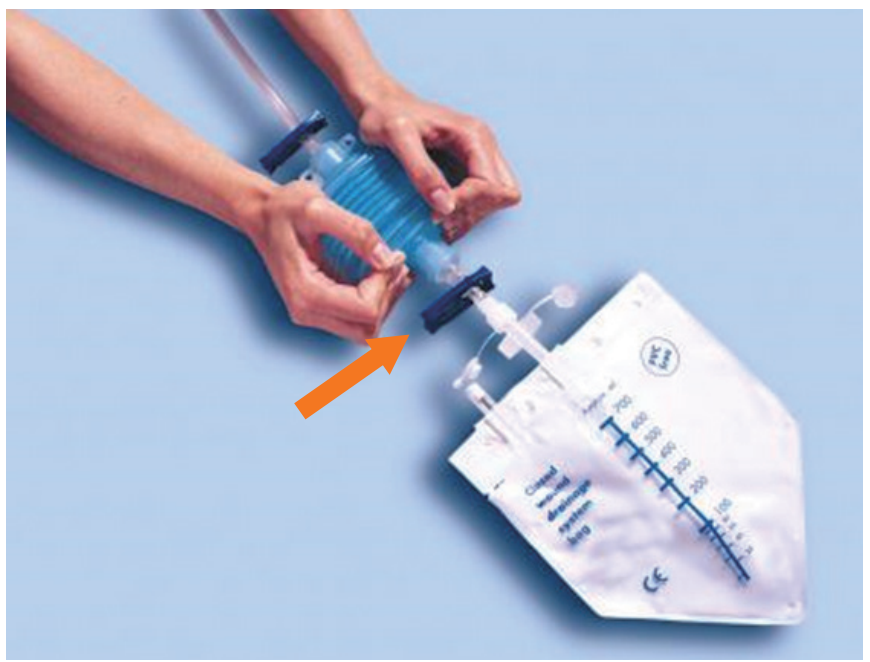

Fig. 1. Drain fluid measurement. Samples are collected by emptying the bellows at the arrow. sion in the study. Patient demographics, surgery details, and postsurgical information were obtained. All patient information was de-identified by the assignment of a unique numeric code, known only to the investigators listed on this project.

Patient vital signs (pulse rate, blood pressure, and temperature) were collected and recorded daily. All adverse events were assessed for any temporal relation to the administered GG flushes. $\mathrm{AL}$ is defined and graded by the criteria set out in the International Study Group document [17].

Blinding of samples for dual-energy computed tomography The drain fluid samples were blinded to the reporting radiologist and scanned as a batch. The results were not available to the treating surgeon.

\section{Analysis and statistics}

All iodine measurements by DECT and statistical analysis was performed using de-identified data. Due to the small sample size in each arm of the study, descriptive statistics were computed for all measurements and displayed graphically using GraphPad Prism 8 (GraphPad, San Diego, CA, USA).

\section{RESULTS}

Twenty-one patients were assessed for inclusion. One patient was ineligible due to an iodine allergy. The first 10 consecutive patients were allocated to receive QID rectal tube flushes with 30$\mathrm{mL}$ saline, as per standard care. Three of these patients (30.0\%) experienced an AL, as diagnosed by CT imaging and clinical course. The following consecutive 10 patients were allocated to receive QID rectal tube flushes with 30-mL GG. One patient (10.0\%) in this group experienced an AL. There was no mortality in either group. All patients underwent a stapled endoluminal anastomosis.

Three patients in the saline flush group, who experienced an $\mathrm{AL}$, were returned to the operating theatre (OT) where they underwent a laparoscopic abdominal lavage and a defunctioning ileostomy was fashioned. One patient in the GG flush group was also returned to OT for a laparoscopic abdominal lavage and defunctioning ileostomy. All ALs were graded as grade C [17]. All 4 patients, who experienced an AL during the study, underwent a standard, single-source CT scan on the day of clinical deterioration and the drains were all confirmed to be in the correct pelvic position. Patients who did not experience an AL did not undergo a CT scan.

The basic observations (temperature, pulse rate, and blood pressure) were monitored and recorded for each patient during the study. No patient was found to have an adverse reaction to the GG during administration. The GG flushes were well tolerated by the patients. There was no prolongation of length of stay or increase in AL rate observed in the GG flush group. The characteristics of the participants are summarized in Table 1. 
Table 1. Characteristics of the 2 study groups; saline and Gastrografin ${ }^{\mathrm{a}}$ flushed rectal tubes

\begin{tabular}{|c|c|c|c|c|}
\hline Characteristic & Overall & Saline flush group & Gastrografin flushes group & P-value \\
\hline No. of patients & 20 & 10 & 10 & \\
\hline Age (yr) & $65.5(54.0-76.3)$ & $67.0(58.0-72.5)$ & $64.0(46.5-82.5)$ & 0.999 \\
\hline Sex & & & & 0.650 \\
\hline Female & $8(40.0)$ & $5(50.0)$ & $3(30.0)$ & \\
\hline Male & $12(60.0)$ & $5(50.0)$ & $7(70.0)$ & \\
\hline Weight (kg) & $82.5(65.0-98.8)$ & $70.0(64.0-84.0)$ & $94.5(74.3-100.0)$ & 0.112 \\
\hline Height (cm) & $172.5(163.3-179.5)$ & $169.5(160.8-177.8)$ & $174.0(169.0-180.0)$ & 0.344 \\
\hline Body mass index $\left(\mathrm{kg} / \mathrm{m}^{2}\right)$ & $25.3(23.9-29.6)$ & $25.2(22.3-26.6)$ & $27.0(24.6-32.7)$ & 0.173 \\
\hline LOS (day) & $5.5(3.0-10.0)$ & $8.5(3.8-10.3)$ & $5.0(3.0-6.3)$ & 0.247 \\
\hline POD of drain removal & $3.5(3.0-7.3)$ & $3.0(3.0-9.3)$ & $4.0(3.0-4.3)$ & 0.871 \\
\hline Anastomotic leak & $4(20.0)$ & $3(30.0)$ & $1(10.0)$ & 0.582 \\
\hline Return to OT & $4(20.0)$ & $3(30.0)$ & $1(10.0)$ & 0.582 \\
\hline Complication (CD classification) & & & & - \\
\hline ।, || & 2 & 1 & 1 & \\
\hline III, IV & 4 & 3 & 1 & \\
\hline Mortality & 0 & 0 & 0 & - \\
\hline
\end{tabular}

Values are presented as number only, median (interquartile range), or number (\%).

LOS, length of hospital stay; POD, postoperative day; OT, operating theatre; CD, Clavien-Dindo.

aBayer Australia Ltd., Pymble, NSW, Australia; registered trademark of the Bayer Group, Germany.

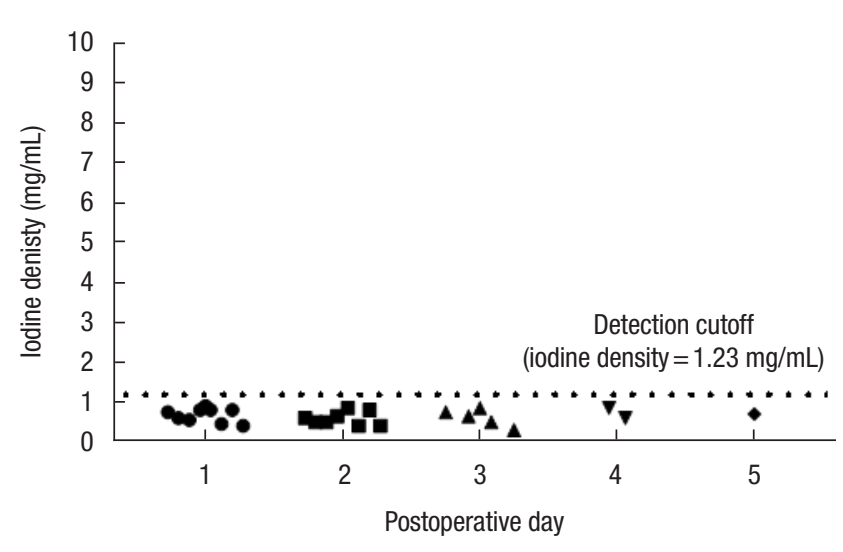

Fig. 2. Iodine density in drain fluid specimens following colorectal surgery in patients not administered Gastrografin (Bayer Australia Ltd., Pymble, NSW, Australia; registered trademark of the Bayer Group, Germany). The dotted line represents the derived detection threshold of 1.23 $\mathrm{mg} / \mathrm{mL}($ mean $+3 \mathrm{SD})$.

In this study, all drain fluid specimens following rectal tubes flushed with saline (saline flush group) resulted in iodine density levels below the detection threshold of $1.23 \mathrm{mg} / \mathrm{mL}$ (Fig. 2).

All rectal tube specimens (intraluminal) from the saline flush group also returned results below the detection threshold (1.23 $\mathrm{mg} / \mathrm{mL}$ iodine), as expected (Fig. 3). All rectal tube specimens,

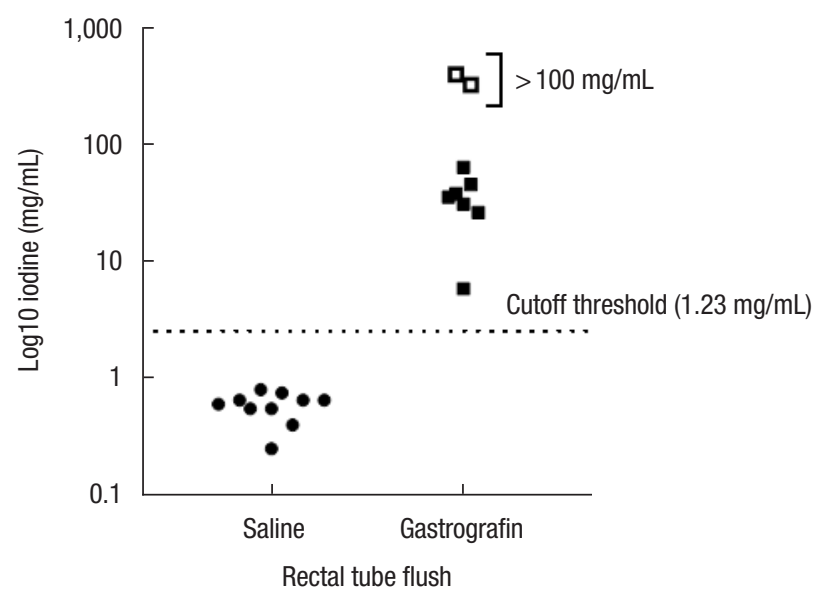

Fig. 3. Measurement of Gastrografin (Bayer Australia Ltd., Pymble, NSW, Australia; registered trademark of the Bayer Group, Germany) by dual-energy computed tomography, in stool of patients administered rectal tube flushes with saline or Gastrografin.

from the GG flush group, returned measurable iodine levels. Thus, high levels of GG were present in the neorectal lumen of this group (Fig. 3). Two samples collected from patients administered GG flushes resulted in iodine levels above the levels measurable by DECT ( $>100 \mathrm{mg} / \mathrm{mL}$ ). This was expected due to beam hardening artifact in the setting of undiluted GG rectal tube 


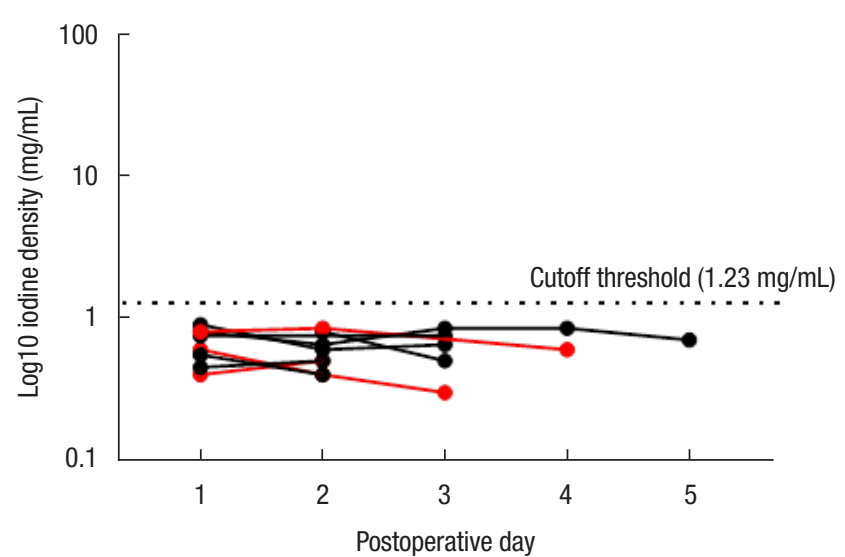

Fig. 4. Log10 iodine density measurements by dual-energy computed tomography in drain fluid of patients administered saline rectal tube flushes. Patients experiencing anastomotic leakage are shown in red, dotted line represents cutoff at $1.23 \mathrm{mg} / \mathrm{mL}$ of iodine density.

flushes. There were no false positives or false negatives.

Drain fluid measurements of GG in the saline flush group showed no increase in iodine in $3 \mathrm{AL}$ patients (Fig. 4).

In this series, there were 3 false-positive readings observed on day 2 and day 3 in the drain fluid of patients who did not experience an AL (Fig. 5). These 3 unexpected results were reevaluated with inductively coupled plasma mass spectrometry (ICP-MS) and iodine measurements concur that the levels measured by DECT were positive for iodine. The results measured by ICP-MS confirm the accuracy of measurement by DECT, but don't explain the unexpected results. Two of these abnormal results returned to levels below the cutoff threshold in patients with subsequent samples, and the other patient had already had their drain removed and been discharged; so no further samples were available for analysis. As all 3 patients were clinically well and their vital observations did not indicate any concerns, they did not undergo any cross-sectional imaging.

A CT scan was performed to investigate 1 patient with a suspected AL in the GG flush group (Fig. 6). This was performed without any routine enteric radiological contrast for the investigation but the extravasated GG flush was readily apparent in the axial slices, highlighting and confirming the diagnosis of AL.

At long-term postsurgical follow-up, all patients who experienced AL during the study had their ileostomy closed and intestinal continuity restored. There were no delayed ALs experienced by study participants.

\section{DISCUSSION}

This study investigates the proof of concept of a novel biomarker

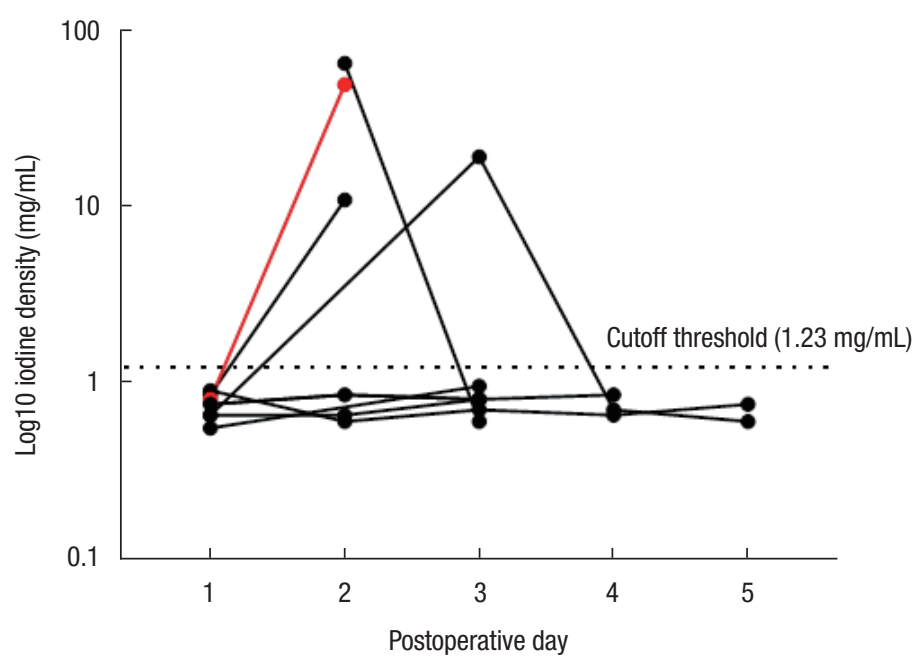

Fig. 5. Log10 iodine density measurements in the drain fluid of patients administered Gastrografin (Bayer Australia Ltd., Pymble, NSW, Australia; registered trademark of the Bayer Group, Germany) rectal tube flushes. One patient who experienced anastomotic leakage is shown in red, dotted line represents detection cutoff at $1.23 \mathrm{mg} / \mathrm{mL}$ iodine density.

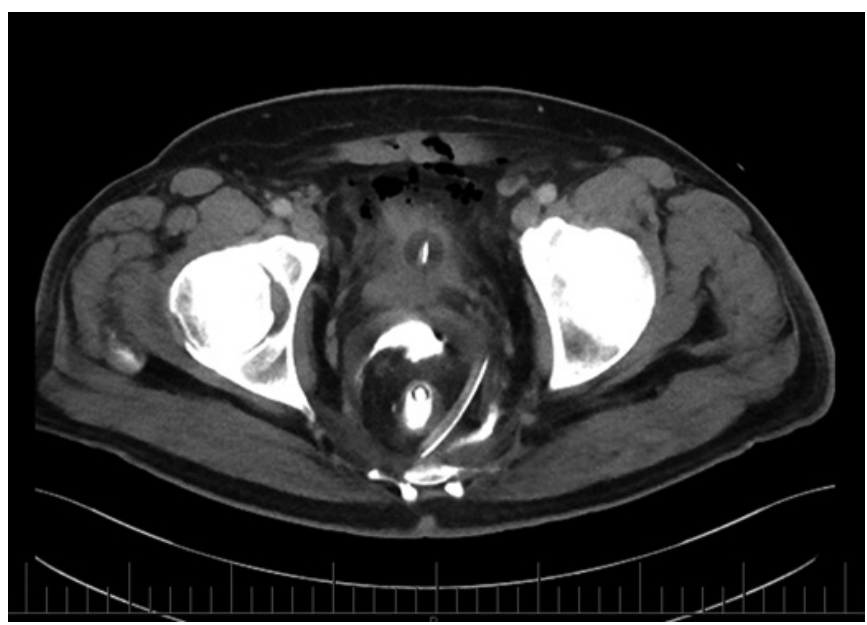

Fig. 6. Axial computed tomography scan to investigate a suspected anastomotic leak in 1 patient in the Gastrografin flush group who suffered an anastomotic leakage. Gastrografin: Bayer Australia Ltd., Pymble, NSW, Australia; registered trademark of the Bayer Group, Germany.

of AL in patients with a low pelvic extraperitoneal colorectal anastomosis and without a diverting loop ileostomy. The flushing of rectal tubes with GG is safe and may be an early biomarker of AL. The measurement of biomarkers using DECT of ex vivo drain fluid avoids any exposure to ionizing radiation to the patients.

Many studies and systematic reviews have been published investigating drain fluid biomarkers $[2,18,19]$. The inflammatory cytokine biomarkers are difficult to interpret as they are measuring 
the environment around the anastomosis and tissue trauma, repair, and remodeling would be expected processes occurring at the operative site in the pelvis. While they are poor biomarkers of $\mathrm{AL}$, they indicate that nonspecific inflammatory processes are occurring early in the postoperative period. A reanalysis of prior trial data by Sammour et al. [20] suggested that peritoneal cytokines can predict clinically important AL on day 1 after surgery.

Pelvic drain use remains controversial [1]. A 2017 review assessed 7 systematic reviews, 1 randomized controlled trial and 7 cohort studies, and concluded that that routine drainage has no significant impact on the rate of colorectal AL but may have a selective utility when the operative field is not dry [21]. The included GRECCAR 5 randomized controlled trial reported no difference in the drained and undrained groups [22]. Earlier reviews have reported a lower AL rate and lower intervention rates in drained patients [23].

The use of rectal tubes is supported by a randomized controlled trial [24] and a subsequent meta-analysis [25]. The authors report that transanal tubes are safe and effective in decreasing the rate of clinically significant ALs and mitigating the clinical consequences of leakage. The meta-analysis included 909 patients, with 1 randomized controlled trial, 1 prospective study, and 2 retrospective studies. A 2018 retrospective study employing propensity score analysis came to similar conclusions [26]. This represents level 1 evidence following the Oxford Centre of Evidence-Based Medicine [27].

In 2010, the International Study Group of Rectal Cancer proposed a definition and grading system for AL [17]. The authors recognized that numerous definitions of AL were found in the literature, and that reported AL rates varied considerably from 3\% to $23 \%$. The agreed definition was "a defect of the intestinal wall at the anastomotic site leading to a communication between the intra- and extraluminal compartments." The thesis underpinning this study is that measurement of an exclusively luminal substance will be detectable in the extraluminal compartment, via a pelvic drain, as an AL occurs. The hope is that early detection of AL may allow investigation and intervention in the window before serious clinical deterioration occurs.

In this Phase 1, stage 2a development study, the DECT was able to detect and quantitate the iodine present in the rectum after regular GG flushes of the rectal tube in all 10 patients administered GG. Furthermore, all values of rectal tube fluid and drain fluid in the 10 patients administered saline flushes were below the cutoff threshold for iodine detection. One patient, who suffered an AL in the GG flush group, showed an elevated GG level on the day of AL and was returned to OT for management. However, 3 other drain fluid specimens returned values above the cutoff threshold from patients who did experience a confirmed AL. Those patients recovered uneventfully and were well at follow-up. Perhaps these patients did suffer a minor AL, detectable in the drain fluid only by DECT, but with no clinically significant effect on the patient or their vital signs. Sample collection error is an- other possibility. A larger study is required to further examine these findings but may prove to be valuable if minor ALs can be identified early in the postoperative course. This may lead to a heightened level of vigilance with more frequent clinical review and lower the threshold for cross-sectional imaging.

In an earlier pilot study [28] from this institution, extravasation of the enzyme amylase was measured in the drain fluid of patients undergoing restorative surgery with an ileal J pouch for ulcerative colitis and without a diverting loop ileostomy. We found very high levels of the enzyme within the lumen of the pouch (over 1,000 times the serum reference range) but levels in the drain fluid, of those patients who did not experience an AL, approximating the serum levels. We concluded that this extravasated intraluminal enzyme rose significantly in drain fluid samples of the patients who did experience an AL. We postulated that drain fluid analysis of extravasated intraluminal substances may allow for the detection of AL before clinical deterioration of the patient.

A limitation of this study is the small sample size. The study was not powered to allow comparison of the control and intervention groups. The aim of safety assessment was delivered by the relatively blunt tool of recording graded complications and mortality. As all eligible patients did not have a defunctioning loop ileostomy, they represent a cohort of patients at a lower risk of AL. The risk factors, considered by the surgeons to arrive at the decision not to defunction the colorectal anastomoses, were not assessed.

The aim of this controlled development study was to demonstrate safety of the proposed study methodology and the feasibility of the DECT measurements of iodine in drain fluid and stool. The clinical application is to employ DECT measurements of iodine in drain fluid as an early biomarker of AL in colorectal surgery with an extraperitoneal anastomosis. Our preliminary findings achieve this objective and show promise of this novel approach but due to small sample size in this stage $2 \mathrm{a}$ development study, further evaluation is required.

In conclusion, this observational stage 2a development study demonstrates the safety of a novel approach to the early detection of AL in low pelvic colorectal anastomoses and would appear to have merit. The technical protocol is feasible and reliable but requires validation in a larger cohort and a multicenter study is planned to investigate the efficacy of GG rectal tube flushes as an early biomarker of AL in low pelvic anastomoses.

\section{CONFLICT OF INTEREST}

No potential conflict of interest relevant to this article was reported.

\section{FUNDING}

None. 


\section{ACKNOWLEDGMENTS}

Tom Snow, Jit Pratap, and John Coucher are to be thanked for their contributions to the initial validation study and with the processing of samples in this study.

\section{REFERENCES}

1. McDermott FD, Heeney A, Kelly ME, Steele RJ, Carlson GL, Winter DC. Systematic review of preoperative, intraoperative and postoperative risk factors for colorectal anastomotic leaks. $\mathrm{Br} J$ Surg 2015;102:462-79.

2. Su’a BU, Mikaere HL, Rahiri JL, Bissett IB, Hill AG. Systematic review of the role of biomarkers in diagnosing anastomotic leakage following colorectal surgery. Br J Surg 2017;104:503-12.

3. Singh PP, Zeng IS, Srinivasa S, Lemanu DP, Connolly AB, Hill AG. Systematic review and meta-analysis of use of serum C-reactive protein levels to predict anastomotic leak after colorectal surgery. Br J Surg 2014;101:339-46.

4. Clark DA, Yeoh E, Edmundson A, Pratap J, Snow T, Solomon M, et al. Gastrografin can be detected in ex vivo biological specimens by dual-energy CT scanning. J Med Imaging Radiat Oncol 2020; 64:634-40.

5. Barkun JS, Aronson JK, Feldman LS, Maddern GJ, Strasberg SM; Balliol Collaboration. Evaluation and stages of surgical innovations. Lancet 2009;374:1089-96.

6. Ergina PL, Cook JA, Blazeby JM, Boutron I, Clavien PA, Reeves $\mathrm{BC}$, et al. Challenges in evaluating surgical innovation. Lancet 2009;374:1097-104.

7. McCulloch P, Altman DG, Campbell WB, Flum DR, Glasziou P, Marshall JC, et al. No surgical innovation without evaluation: the IDEAL recommendations. Lancet 2009;374:1105-12.

8. Rogers WA, Hutchison K, McNair A. Ethical issues across the IDEAL stages of surgical innovation. Ann Surg 2019;269:229-233.

9. McCulloch P, Cook JA, Altman DG, Heneghan C, Diener MK; IDEAL Group. IDEAL framework for surgical innovation 1: the idea and development stages. BMJ 2013;346:f3012.

10. Dindo D, Demartines N, Clavien PA. Classification of surgical complications: a new proposal with evaluation in a cohort of 6336 patients and results of a survey. Ann Surg 2004;240:205-13.

11. Therapeutic Goods Administration (TGA) of Department of Health, Australian Government. ARTG ID 10684: Gastrografin oral liquid bottle [Internet]. Woden Valley (ACT): TGA; c2021 [cited $2021 \mathrm{Feb} 8$ ]. Available from: https://search.tga.gov.au/s/ search.html? collection=tga-artg\&profile=record\&meta_i $=10684$

12. Flores-Funes D, Campillo-Soto Á, Pellicer-Franco E, Aguayo-Albasini JL. The use of coffee, chewing-gum and gastrograffin in the management of postoperative ileus: a review of current evidence. Cir Esp 2016;94:495-501.

13. Habib K, Gupta A, White D, Mazari FA, Wilson TR. Utility of contrast enema to assess anastomotic integrity and the natural history of radiological leaks after low rectal surgery: systematic review and meta-analysis. Int J Colorectal Dis 2015;30:1007-14.

14. Biondo S, Miquel J, Espin-Basany E, Sanchez JL, Golda T, FerrerArtola AM, et al. A double-blinded randomized clinical study on the therapeutic effect of Gastrografin in prolonged postoperative ileus after elective colorectal surgery. World J Surg 2016;40:20614.

15. Chintamani RB. Randomized clinical study of Gastrografin administration in patients with adhesive small bowel obstruction (Br J Surg 2003; 90: 542-546). Br J Surg 2003;90:1165.

16. Aran S, Shaqdan KW, Abujudeh HH. Dual-energy computed tomography (DECT) in emergency radiology: basic principles, techniques, and limitations. Emerg Radiol 2014;21:391-405.

17. Kulu Y, Ulrich A, Bruckner T, Contin P, Welsch T, Rahbari NN, et al. Validation of the International Study Group of Rectal Cancer definition and severity grading of anastomotic leakage. Surgery 2013;153:753-61.

18. Hirst NA, Tiernan JP, Millner PA, Jayne DG. Systematic review of methods to predict and detect anastomotic leakage in colorectal surgery. Colorectal Dis 2014;16:95-109.

19. Sparreboom CL, Wu Z, Dereci A, Boersema GS, Menon AG, Ji J, et al. Cytokines as early markers of colorectal anastomotic leakage: a systematic review and meta-analysis. Gastroenterol Res Pract 2016;2016:3786418.

20. Sammour T, Singh PP, Zargar-Shoshtari K, Su’a B, Hill AG. Peritoneal cytokine levels can predict anastomotic leak on the first postoperative day. Dis Colon Rectum 2016;59:551-6.

21. Emile SH, Abd El-Hamed TM. Routine drainage of colorectal anastomoses: an evidence-based review of the current literature. Gastroenterol Res Pract 2017;2017:6253898.

22. Denost Q, Rouanet P, Faucheron JL, Panis Y, Meunier B, Cotte E, et al. To drain or not to drain infraperitoneal anastomosis after rectal excision for cancer: the GRECCAR 5 randomized trial. Ann Surg 2017;265:474-80.

23. Rondelli F, Bugiantella W, Vedovati MC, Balzarotti R, Avenia N, Mariani E, et al. To drain or not to drain extraperitoneal colorectal anastomosis?: a systematic review and meta-analysis. Colorectal Dis 2014;16:O35-42.

24. Xiao L, Zhang WB, Jiang PC, Bu XF, Yan Q, Li H, et al. Can transanal tube placement after anterior resection for rectal carcinoma reduce anastomotic leakage rate?: a single-institution prospective randomized study. World J Surg 2011;35:1367-77.

25. Shigeta K, Okabayashi K, Baba H, Hasegawa H, Tsuruta M, Yamafuji $\mathrm{K}$, et al. A meta-analysis of the use of a transanal drainage tube to prevent anastomotic leakage after anterior resection by double-stapling technique for rectal cancer. Surg Endosc 2016;30: 543-50.

26. Yang CS, Choi GS, Park JS, Park SY, Kim HJ, Choi JI, et al. Rectal tube drainage reduces major anastomotic leakage after minimally invasive rectal cancer surgery. Colorectal Dis 2016;18:0445-52.

27. OCEBM Levels of Evidence Working Group. The Oxford 2011 levels of evidence [Internet]. Oxford (UK): Oxford Centre for Evidence-Based Medicine; 2011 [cited 2021 Feb 8]. Available from: 
http://www.cebm.net/wp-content/uploads/2014/06/CEBM-Levels-of-Evidence-2.1.pdf

28. Clark DA, Cuda T, Riddell A, Radford-Smith G, Solomon M.
Drain fluid amylase as a sensitive biomarker for the early detection of anastomotic leakage in ileal pouch surgery. Colorectal Dis 2019;21:460-4. 


\section{Appendix 1:}

\section{Standard operating protocol: \\ Measurement of Gastrografin in surgical drain fluid and stool by Dual Energy CT (DECT)}

\section{Background}

The benefit of DECT is achieved via its ability to distinguish between materials based on their spectral properties; a material's capacity to attenuate $\mathrm{x}$-ray photons at different photon energies. Materials interact with $\mathrm{x}$-ray photons in several ways including the Compton effect and the photoelectric effect ${ }^{1}$. DECT operates on the principle that two different $\mathrm{x}$-ray spectra will cause a material to absorb $\mathrm{x}$-ray photons differently according to these interactions. This particularly applies for the photoelectric effect which is dependent upon a material's properties, such as the atomic number, electron density and K-edge electron energy. The widely used CT contrast agent iodine has a high atomic number $(Z=53)$ which results in a strong photoelectric effect. This photoelectric effect and spectral behaviour can be measured by DECT and can be used to detect and quantify iodine containing substances, such as Gastrografin².

\section{DECT scan protocol and sample measurement}

Daily $10 \mathrm{~mL}$ samples of drain fluid were collected into $50 \mathrm{~mL}$ sterile jars and stored at room temperature. All samples were scanned via the same dual source CT system (Somatom Force, Siemens Medical Solutions, Forchheim, Germany) in the same radiology department by the same senior CT radiographer. The samples were placed flat onto the CT table and evenly spaced by $10 \mathrm{~cm}$. The samples were scanned within 4 weeks of sample collection.

The dual energy protocol with acquisition parameters of $80 \mathrm{kV}$ and Qref mAs of $100 \mathrm{mAs}$ on the A tube and sn150 kV and QRef mAs of $67 \mathrm{mAs}$ on the B tube were used to scan each sample. To deliver a constant radiation dose for all scans, no dose modulation was utilised. Each detector was collimated to $128 \times 0.6 \mathrm{~mm}$ with a flying focal spot, and a pitch of 0.7 was applied. Images were reconstructed with a dedicated Br40 reconstruction algorithm. Slice thickness and increment were $1.0 \mathrm{~mm}$ and $0.7 \mathrm{~mm}$, respectively. Two individual stacks of images for each detector $(80 \mathrm{kV}$ and sn150 kV images) and a DE mixed images were reconstructed. The latter will contain weighted information from both detectors with a weighting factor of 0.6 (60\% from the $80 \mathrm{kV}$ scan and $40 \%$ from the sn $150 \mathrm{kV}$ scan) thus approximating regular $120 \mathrm{kV}$ images.

After reconstruction, images were transferred to the same workstation with dedicated commercial post-processing software (Syngo Dual Energy, Siemens Medical Solutions, Forchheim, Germany). The dual energy datasets were analysed using the LiverVNC software where iodine density and $\mathrm{HU}$ were measured for each sample without any manual adjustments to the algorithm.

Two separate region of interest (ROI) measurements were used to measure the $\mathrm{HU}$ and iodine density $(\mathrm{mg} / \mathrm{mL})$ of each sample. The ROI measurements were obtained via coronal reformats to the right and left of the solution midline, each ROI measuring $10 \mathrm{~mm}^{2}$. All measurements were performed by the same radiologist who remained blinded to the samples.

Samples were re-scanned twice during the course of the study, and if significant discrepancies between results were present, the solutions would be rescanned twice more. The two individual $\mathrm{HU}$ and iodine concentration results for each sample was recorded as per the data collection described in the protocol.

\section{Preliminary studies of iodine measurement by DECT}

Our preliminary in vitro and ex vivo studies have contributed to the development of DECT protocol (Appendix 1) to detect the presence of GG in a sample solution of sterile water or surgical drain fluid. The lower limit of detection of a solution containing GG was at a concentration of $0.097 \%$, which correlated to an iodine density of $>1 \mathrm{mg} / \mathrm{mL}$. The upper limit of the true negative levels of GG measured in drain fluid by DECT was derived by calculating the mean $(+3 \mathrm{SD})$ iodine density $(\mathrm{mg} / \mathrm{mL})$ in GG negative samples. We have established measurements of iodine density above $1.2 \mathrm{mg} / \mathrm{mL}$ to represent a positive result for the presence of GG in a solution measured by DECT. The methodology for the quantitation of GG in biological fluids has been reported in a submitted technical paper looking at serial dilutions and stability of GG in vitro.

'Coursey CA, Nelson RC, Boll DT, Paulson EK, Ho LM, Neville AM, et al. Dual-energy multidetector CT: how does it work, what can it tell us, and when can we use it in abdominopelvic imaging? Radiographics 2010;30:1037-55.

${ }^{2}$ Johnson TR. Dual-energy CT: general principles. AJR Am J Roentgenol 2012;199(5 Suppl):S3-8. 